\title{
SCIENTIFIC CORRESPONDENCE
}

\section{An evaluation of the administration of sub-Tenon local anaesthesia by a nurse practitioner}

\author{
H Waterman, S Mayer, M J Lavin, A F Spencer, C Waterman
}

Br J Ophthalmol 2002;86:524-526

Aim: To evaluate the administration of sub-Tenon local anaesthesia (LA) by a nurse practitioner.

Methods: 106 consecutive patients listed for cataract surgery were given sub-Tenon's anaesthesia by a nurse practitioner. The surgical procedure was performed within 15 minutes of the administration of the LA. Pain, state and adequacy of anaesthesia, appearance of the eye, and patient anxiety were measured. Patients' experiences of this new nursing role were gained through interview. Results: At the beginning of surgery, few patients showed eye movement in any of the four quadrants and the surgeons reported that akinesia was inadequate in only seven $(7.1 \%)$ cases. Three patients $(3.3 \%)$ gave a pain score of 3 out of 10 or above during surgery whereas the surgeons assessed the pain relief to be inadequate in eight $(8.1 \%)$ cases. Five $(5.3 \%)$ patients required a top up of anaesthesia and $51(39.8 \%)$ patients developed conjunctival chemosis in two or more quadrants. Overall, patients' comments indicate that they were pleased with the new service.

Conclusion: The results suggest that nurse practitioner delivered sub-Tenon LA is an effective and safe method of LA administration for cataract surgery.

s n a national survey, it was found that $70 \%$ of intraocular surgery is performed using local anaesthesia (LA), 24.2\% using general anaesthesia (GA), and 5\% using LA plus sedation. ${ }^{1}$ The same survey reports that a small proportion $(6.7 \%)$ of LAs were by sub-Tenon injection. Sub-Tenon anaesthesia has been favourably compared to retrobulbar and peribulbar anaesthesia. ${ }^{2-6}$ Recently, there has been increasing interest in sub-Tenon and topical LA techniques at Manchester Royal Eye Hospital. One hundred and six consecutive patients (106 eyes) were surveyed between June and August 1999 in order to evaluate the nursing role in the administration of sub-Tenon LA. The objectives of the study were to assess pain, state and adequacy of anaesthesia, appearance of the eye, and patients' experiences.

\section{METHODS}

In 1998, a successful application was made to the clinical director at MREH to educate and train a staff nurse (SM) to administer sub-Tenon LA. The main purpose of this nursing development was to improve the patient experience of LA and to reduce times between patient cases. ${ }^{7}$ From the beginning of November 1998 to the end of January 2001, SM had performed over 2500 sub-Tenon injections.

All patients were cannulated by the nurse to ensure intravenous access. Monitoring of pulse oximetry and electrocardiogram were performed either by SM or the assisting nurse. Firstly, all patients received three drops of bupivacaine topically to the cornea and conjunctiva. Then patients were asked to look up and outward temporally to allow exposure of the inferonasal quadrant of the eye. Using a pair of Moorfields forceps, the conjunctiva was picked up approximately $10 \mathrm{~mm}$ from the limbus and a small nick incision was made using blunt scissors. A Southampton cannula (20 gauge) was then introduced and $0.5 \mathrm{ml}$ of local anaesthetic applied to separate the conjunctiva and Tenon's fascia. The scissors were then re-introduced to create an opening in the anterior Tenon's fascia down to bare sclera. The cannula was then reintroduced and guided along a path following the contour of the globe until the tip was past the posterior of the equator of the globe. Slow delivery of $3 \mathrm{ml}$ of local anaesthetic was then performed.

A group protocol indicated the type of local anaesthetic agent to be used. All patients received hyaluronidase ( 1500 IU) together with either prilocaine $3 \%$ with octapressin or prilocaine $4 \%$ or lignocaine $2 \%$ with $0.5 \%$ Marcain (bupivacaine). ( Since this study was completed, prilocaine 3\% with octopressin has been implicated in serious complications of regional anaesthesia for ophthalmic surgery (Astra Zeneca January 2000, reference 6058/CM/SH/ZA/sf). We no longer use prilocaine $3 \%$ with octapressin preferring instead prilocaine $4 \%$.) The eye was then prepared for surgery using povidoneiodine to the upper and lower fornix, conjunctiva, and eyelids.

Local research ethics committee approval was sought but deemed not to be required. No patients refused to take part. Data were collected systematically on several variables: age, sex, type of surgical procedure, anaesthetic agents, number of anaesthetic drops, volume of anaesthetic drops and top up of anaesthetic agent if required. Pain scores were collected using a 11 item numerical rating scale, in which 0 represents "no pain" and 10 "worst pain imaginable." Akinesia, conjunctival chemosis, and haemorrhage were measured according to number of quadrants involved. ${ }^{8}$ Rotation of superior oblique muscle and eyelid movement were assessed by presence or absence. Perceived adequacy of anaesthesia was measured by surgeons' "Yes" or "No" assessment of pain relief, and whether there was akinesia inadequacy, vitreous bulge, or a shallow anterior chamber.

Anxiety was selected as a variable for measurement because of its association with heightened feelings of pain. Patient anxiety was measured by the Amsterdam preoperative anxiety and information scale. ${ }^{9}$ This scale was chosen because it was quick to administer, having four questions only. Each question has a five item scale which is used to gain the degree of anxiety. Total scores therefore range from 4 to 20. Anxiety is categorised as moderate when the total score ranges from 11 to 15 and severe when the total score is 16 to 20 .

Quantitative data were entered into Statistical Package for Social Sciences and analysed using descriptive statistics and $\chi^{2}$ tests.

Ten patients were selected for a semistructured interview, which was undertaken away from the theatre after patients had recovered but before they were discharged home. The interviews were carried out on consecutive patients from three theatre lists chosen by convenience. 


\begin{tabular}{|c|c|c|c|c|}
\hline Pain score & $\begin{array}{l}\text { Administration of local } \\
\text { anaesthetic }\end{array}$ & I Operation & $\begin{array}{l}\text { Subconjunctival } \\
\text { injection }\end{array}$ & After surgery \\
\hline 0 & 37 (40.7\%) & $69(77.5 \%)$ & 70 (70.6\%) & 76 (89.4\%) \\
\hline 1 & $19(20.9 \%)$ & $12(13.5 \%)$ & $10(11 \%)$ & $7(8.2 \%)$ \\
\hline 2 & $19(20.9 \%)$ & $5(5.6 \%)$ & $6(6.6 \%)$ & $1(1.2 \%)$ \\
\hline 3 & $7(7.7 \%)$ & $1(1.1 \%)$ & $5(5.5 \%)$ & - \\
\hline 4 & $2(2.2 \%)$ & $1(1.1 \%)$ & - & $1(1.2 \%)$ \\
\hline 5 & $4(4.4 \%)$ & - & - & - \\
\hline 6 & $1(1.1 \%)$ & $1(1.1 \%)$ & - & - \\
\hline 7 & - & - & - & - \\
\hline 8 & - & - & - & - \\
\hline 9 & - & - & - & - \\
\hline 10 & - & - & - & - \\
\hline Total & 91 (100\%) & $89(100 \%)$ & 91 (100\%) & 85 (100\%) \\
\hline
\end{tabular}

\begin{tabular}{|c|c|c|c|c|}
\hline Movement & Elevation & Depression & Abduction & Adduction \\
\hline \multicolumn{5}{|c|}{ At start of surgery } \\
\hline None & $64(62.7 \%)$ & $66(65.3 \%)$ & 71 (72.4\%) & $71(72.4 \%)$ \\
\hline Slight & $30(29.4 \%)$ & $28(27.7 \%)$ & $18(18.4 \%)$ & $20(20.4 \%)$ \\
\hline Moderate & $8(7.8 \%)$ & $6(5.9 \%)$ & $8(8.2 \%)$ & $5(5.1 \%)$ \\
\hline Complete & - & $1(1 \%)$ & $1(1 \%)$ & $2(2 \%)$ \\
\hline Total & $102(100 \%)$ & $101(100 \%)$ & $98(100 \%)$ & $98(100 \%)$ \\
\hline \multicolumn{5}{|c|}{ At end of surgery } \\
\hline None & 72 (72.7\%) & 73 (73.7\%) & 75 (77.3\%) & 74 (77.9\%) \\
\hline Slight & $17(17.2 \%)$ & $17(17.2 \%)$ & 15 (15.5\%) & $15(15.8 \%)$ \\
\hline Moderate & $9(9.1 \%)$ & $5(5.1 \%)$ & $6(6.2 \%)$ & $3(3.2 \%)$ \\
\hline Complete & $1(1 \%)$ & $4(4 \%)$ & $1(1 \%)$ & $3(3.2 \%)$ \\
\hline Total & $99(100 \%)$ & $99(100 \%)$ & $97(100 \%)$ & $95(100 \%)$ \\
\hline
\end{tabular}

\section{RESULTS}

The age range of patients was 32-98 years, mean 74.7 (SD $11.3)$ years. There were $60(57.7 \%)$ female patients. Patients undergoing phacoemulsification and insertion of intraocular lens formed the largest group $(n=99,94.3 \%)$. Two $(1.9 \%)$ patients underwent phacoemulsification and insertion of intraocular lens plus trabeculectomy, two (1.9\%) patients had extracapsular cataract extraction, and two (1.9\%) other patients had cyclodiode.

The minimum number of bupivacaine drops given were three in $101(97.1 \%)$ cases and the maximum number were five in one ( $1 \%)$ case. The majority of patients $(n=98,95.1 \%)$ received $3 \mathrm{ml}$ of local anaesthetic; however, two (1.9\%) and three $(2.9 \%)$ patients needed 4 and $5 \mathrm{ml}$ respectively. Five $(5.3 \%)$ patients required a top up of local anaesthetic of which three were topical, one was by injection, and one unknown. The most frequently $(n=98,96.1 \%)$ used local anaesthetic was prilocaine $3 \%$ plus octapressin $2.2 \mathrm{ml}$ or prilocaine $4 \%$. Lignocaine $2 \%$ with Marcain were given in four $(3.9 \%)$ cases.

The distribution of pain scores at different time points throughout the procedure are shown in Table 1. A small number, seven $(7.7 \%)$, patients reported pain scores between 4-6 at the time of the sub-Tenon's injection. A larger proportion of patients stated that they felt a limited degree of pain during surgery $(\mathrm{n}=20,22.5 \%)$ and during the subconjunctival injection $(n=21,23.1 \%)$. The majority of patients, 84 (98.8\%), had pain scores between 0-2 immediately after surgery.

Akinesia was assessed in four quadrants at the commencement of surgery (Table 2 ).

Further calculations revealed that in 55 patients there was no movement of the eye in any direction. Movement of patients' eyes was also assessed at the end of surgery (Table 2). The numbers of patients, 65, with no movement in any quad- rant of the eye appears to have risen slightly over the course of the surgery.

Under a half of patients $(n=37,42.5 \%)$ experienced rotation of the superior oblique muscle. Lid movement was observed in $63(61.2 \%)$ and $60(59.4 \%)$ patients at the commencement and end of surgery respectively.

Conjunctival chemosis occurred in two or more quadrants in $41(39.8 \%)$ patients. A majority of patients $(n=72,69.9 \%)$ had either no or one quadrant of subconjunctival haemorrhage.

Surgeons were asked their opinion as to whether the anaesthesia was adequate with regard to (a) patients' pain; (b) akinesia; (c) vitreous bulge; (d) shallow anterior chamber. On eight $(8.1 \%)$ occasions surgeons thought the patients' pain relief was inadequate. In seven $(7.1 \%)$ cases, surgeons stated that akinesia was inadequate. Vitreous bulge was identified in four $(4.1 \%)$ patients. A shallow anterior chamber was noted by surgeons in nine $(9.1 \%)$ cases.

Just over a third $(n=37,35.2 \%)$ of patients expressed no anxiety. A small proportion of patients $(n=9,8.6 \%)$ had moderate or severe anxiety.

No patients had any systemic reaction to the LA. (Interestingly, an audit of a larger consecutive sample of patients $(n=2500)$ that had received sub-Tenon injection by SM, revealed that four $(0.17 \%)$ patients had an adverse systemic reaction of which three were vasovagal reactions and the other an allergic response; none was considered life threatening.)

Patients appeared to be very satisfied with the local anaesthetic being administered by a nurse, as one patient stated: "The nurse seemed very competent. It couldn't have been done better by anyone else" (Interview 7). The patients emphasised the nurses' ability to make patients feel more relaxed: "The nurse has more time to put you at ease than a 
doctor" (Interview 7). Patients generally considered that provided the professional concerned was trained, it did not matter to them who gave the LA: "Anyone who is trained to do it, doesn't make any difference to me" (Interview 6).

\section{DISCUSSION}

As discussed, $3 \mathrm{ml}$ of anaesthetic was administered to the majority patients in our study only five needing a top up. Pain scores were at the lower end of the scale in our study; the large majority $(96.7 \%)$ of patients scoring 2 or lower during and after surgery. Akinesia appears to have been achieved in the majority of cases as per surgeon assessment.

No serious systemic side effects have been reported since SM began her new role; however, she has been trained in advanced life support skills and an anaesthetist is on duty in the operating department should an emergency occur. All patients are preassessed and any coexisting systemic conditions are identified and treated by appropriately trained personnel. SM assesses each patient before administering the LA and will refer patients to medical staff if thought necessary.

In general, the cosmetic effect of subconjunctival haemorrhage is a frequent reason given for not employing this LA technique. Stevens used adrenaline $0.1 \%$ immediately before subconjunctival incision to minimise haemorrhage and found that $32 \%$ of his sample had a subconjunctival haemorrhage involving one or more quadrant. ${ }^{3}$ We are investigating the possibility of using cautery to prevent haemorrhage.

Overall, the results suggest that a nurse practitioner can administer uncomplicated sub-Tenon local anaesthesia safely and effectively with similar effect as other health professionals. There was a high degree of patient acceptance for this mode of LA administration.

\section{ACKNOWLEDGEMENT}

We would like to thank all patients and staff who supported and helped with the study.

\section{Authors' affiliations}

H Waterman, School of Nursing, Midwifery and Health Visiting, University of Manchester

S Mayer, M J Lavin, A F Spencer, Manchester Royal Eye Hospital

C Waterman, Manchester School of Management, UMIST

Correspondence to: Dr Heather Waterman, School of Nursing, Midwifery and Health Visiting, Coupland III, University of Manchester, Oxford Road, Manchester M13 9PL, UK; heather.waterman@man.ac.uk

Accepted for publication 28 November 2001

\section{REFERENCES}

1 Eke T, Thompson JR. The national survey of local anaesthesia for ocular surgery. I. Survey methodology and current practice. Eye 1999;13: 189-95.

2 Greenbaum S, Parabulbar anaesthesia. Am J Ophthalmol 1992; $114: 776$.

3 Stevens JD. A new local anaesthesia technique for cataract extraction by one quadrant sub-Tenon infiltration. Br J Ophthalmol 1992;76:670-4 4 Briggs MC, Beck SA, Esakowitz L. Sub-Tenon versus peribulbar anaesthesia for cataract surgery. Eye 1997;11:639-43.

5 Eke T, Thompson JR. The national survey of local anaesthesia for ocular surgery. II. Safety profiles of local anaesthesia techniques. Eye 1999;13:196-204.

6 Tokuda, Oshika T, Amano S, et al. Anaesthetic dose and analgesic effects of sub-Tenon anaesthesia in cataract surgery. J Cataract Refract Surg 1999:25:1250-3.

7 Mayer S, Waterman H, Grabham J. Developing a nursing role for the new millenium. Ophthalmic Nursing 2000;3:22-3.

8 Roman SJ, Sit DAC, Boureau CM, et al. Sub-Tenon anaesthesia: an efficient and safe technique. Br J Ophthalmol 1997;81:673-6.

9 Moermans N, Frits SAM, Van Dam Muller M, et al. Amsterdam pre-operative Anxiety and Information Scale (APAIS). Anesth Anal 1996;82:445-51.

\section{Video Reports (www.bjophthalmol.com)}

- Capsule staining and mature cataracts: a comparison of indocyanine green and trypan blue dyes. D F Chang

- Pearls for implanting the Staar toric IOL. D F Chang

- An intraocular steroid delivery system for cataract surgery. D F Chang

- Evaluation of leucocyte dynamics in mouse retinal circulation with scanning laser ophthalmoscopy. Heping Xu, A Manivannan, Garry Daniels, Janet Liversidge, Peter F Sharp, John V Forrester, Isabel J Crane

- Dipetalonema reconditum in the human eye. T Huynh, J Thean, R Maini 